\title{
Optical Materials
}

\section{Preface: Photoluminescence in rare earths: Photonic materials and devices}

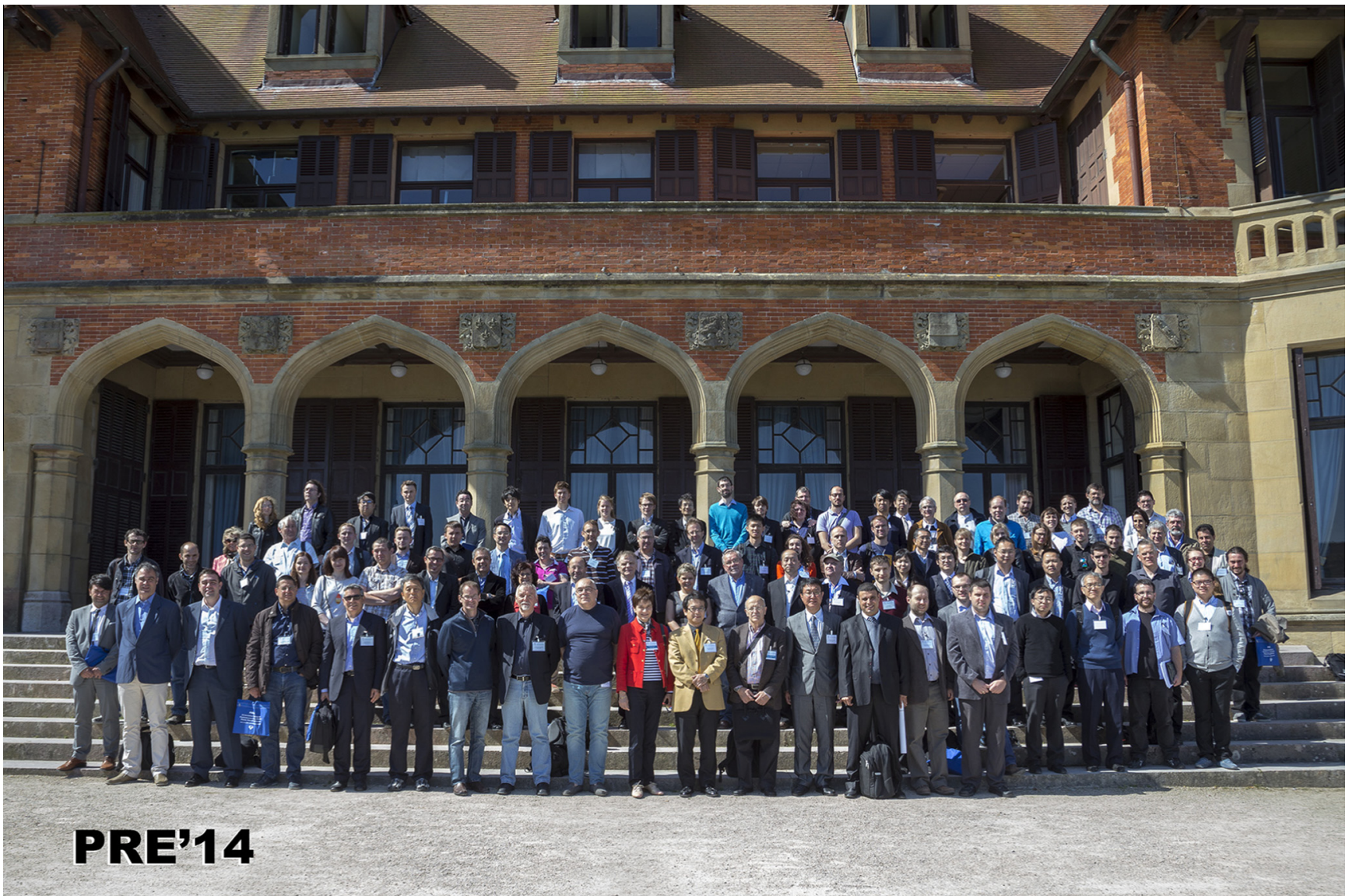

Nowadays the impact of optics technologies can be recognized by the downstream of innovations and new applications that are enabled. They have found applications in manufacturing, health care, information technology, entertainment, and communications. As to the materials, rare earths (RE) represent a strategic group playing a very important role due to the variety of their photoluminescence and allowed excitation procedures. Some applications in the field of biophotonics include, among others, bioimaging, biosensing, optical diagnostics, and light based therapies which hold a great promise for the future. Other important ones can be found in the field of energy harvesting and telecommunications where down and upconversion energy processes can be used to improve the efficiency of solar cells and generate new light sources.

The Workshop on Photoluminescence in Rare Earths: Photonic Materials and Devices (PRE) has been held in Italy (Trento and Florence) three times and in Japan (Kyoto) once. Following the good tradition of the previous workshops, (PRE'05, PRE'07, PRE'10, and PRE'12), the 5th PRE provided a forum for material scientists, 
chemists, and physicists for critical assessment and evaluation of recent developments and future trends in the field of photonic materials and devices based on RE ions.

The PRE'14 workshop, sponsored by the Basque Country Government, Basque Country University UPV-EHU, Donostia International Physics Center, International Commission on Glass, and the Italian Committee Fotonica.It, was organized at the "Palacio Miramar" in San Sebastian, Spain, in May 13-16, 2014.

The Workshop included 15 invited (one plenary) talks, 52 oral talks, and 55 posters and attracted over 110 participants from 23 countries. A special session was organized to honour the 70th birthday of Prof. Giancarlo Righini (former Director of the National Department of Materials and Devices at the National Research Council of Italy, CNR, and since 2012 Director of the Enrico Fermi Centre, in Rome) for his achievements in the field of photonics and quantum electronics. It is worth remarking that the International Workshop on Photoluminescence in Rare Earths: Photonic Materials and Devices (PRE) was initiated and promoted by himself together with Dr. Maurizio Ferrari.

The hot topics during the workshop included ceramic lasers for energy, novel phosphor materials for solid-state lighting, rareearth doped nanoparticles for bio-imaging and therapy, luminescence and scintillation properties of materials, phosphors for light converters, photocatalytic activity, sensors, optical amplification etc.

The present Special Issue provides significant examples of the above mentioned topics and give a broad scope of the conference goals.
The success of 5th PRE resulted from the efforts of many people. We wish to express our special thanks to all the members of the Scientific and Organizing Committees who very much helped us to assemble a valuable technical program and to smoothly run the Workshop.

The edition of this special issue was possible thanks to the collaboration of all the authors who submitted their latest findings, and to the efforts of the referees whose reviewing care made possible the high quality of the published papers.

Finally, we wish to thank the Optical Materials Editor-in-Chief, Prof. Georges Boulon, who offered us the possibility of this Special Issue, and the Elsevier staff for their help.

We look forward to the next series, PRE'16. Prof. John Ballato will welcome the participants in Greenville, South Caroline, in 2016.

Rolindes Balda Joaquín Fernández University of the Basque Country UPV-EHU, Spain Materials Physics Center CSIC-UPV/EHU, Spain

Donostia International Physics Center, Spain E-mail addresses: rolindes.balda@ehu.es (R. Balda), joaquin.fernandez@ehu.es (J. Fernández)

Maurizio Ferrari CNR-IFN, Trento, Italy E-mail addresses: mferrari@science.unitn.it 